\title{
Special representations of nilpotent Lie groups and the associated Poisson representations of current groups
}

\author{
A. M. Vershik 1 ) and M. I. Graev 2 \\ To the memory of our teacher and coauthor, the great Israel Gelfand
}

\section{Contents}

1. Canonical families and the associated special representations of groups

2. The semidirect products $G=\mathbb{R}_{+}^{*} \wedge G_{0}$ and their special representations

2

3. Special representations of nilpotent Lie groups and their one-dimensional extensions

6

4. The special representation of the group $\mathbb{R}_{+}^{*} \wedge N$ associated with the regular representation of $N$

5. The case of the Heisenberg group

10

6. The Poisson representations of current groups $N^{X}$, where $N$ is a graded nilpotent Lie group

7. The extension of the representation $U$ of the group $N^{X}$ to a representation of the group $G^{X}$, where $G=\mathbb{R}_{+}^{*} \wedge N$

8. The Poisson representation of the current group $P^{X}=\left(\mathbb{R}_{+}^{*}\right)^{X} \wedge N^{X}$, where $N$ is the Heisenberg group of order $2 n-1$, and its extension to a representation of the current group $U(n, 1)^{X}$

References

The goal of this paper is to give a short version of the new model of the representation of the current groups with a semisimple Lie group like $(U(n, 1))^{X},(O(n, 1))^{X}$. In the eạrlier papers of 70-80-th with I.M.Gelfand who had posed the problem about irreducible representations of the current group for $S L(2, R)$, we have used a Fock space ([9, 3]) simi- Jar to Araki's formalism ([1]). The construction of the Fock unitary representation of the current group $G^{X}$, where $G$ is an arbitrary locally compact group, is based on a special Trepresentation of the original group $G$, i.e., a unitary representation of $G$ with nontrivial 1-cohomology (see, e.g., [1, 9]). Some groups, including a part of semisimple rank 1 Lie groups, have irreducible special unitary representations; see [10, 3] and the references in [10]. A necessary condition for this is that the identity representation is not isolated

Key words and phrases. Current group, canonical representation, special representation.

1) Partially supported by the RFBR grants 11-01-00677-a, 11-01-12092-ofi-m, 10-01-90411-ukr-a.

${ }^{2)}$ Partially supported by the RFBR grant 10-01-00041a. 
in the space of irreducible representations (i.e., the absence of Kazhdan's property), see [12, 2]. The representations of the current groups $G^{X}$ corresponding to irreducible special representations are also irreducible.

However after our papers (see [10, 11] and previous) became clear that the crucial role in the constructions play nilpotent subgroups and its one-dimensional extensions. In the same time for commutative and nilpotent groups $G$, only the identity representation is special, and the corresponding 1-cohomology group is just the group of characters of $G$ (i.e., the group of homomorphisms from $G$ to $\mathbb{T}$ ). Nevertheless, there is a class of groups, including Lie groups, that have no irreducible special representations, but have reducible special representations. These representations also give rise to construct the irreducible representations of current groups in Fock spaces. In this paper, we confine ourselves to simply connected nilpotent Lie groups on which there is a nontrivial action of the group of automorphisms $\mathbb{R}_{+}^{*}$. In particular, this class contains all connected, simply connected abelian and metabelian Lie groups, including the Heisenberg group and its generalizations. This choice is justified by the fact that irreducible special representations of semisimple Lie groups, if they do exist, in fact come from reducible special representations of certain nilpotent subgroups. This reduction to nilpotent subgroups is useful also in other cases. On the other hand, our construction for nilpotent groups is extremely simple and reduces just to the construction of semidirect products.

Starting from an arbitrary unitary representation $T$ of a nilpotent group $N$ of the form described above, we construct a special (reducible) representation $\widetilde{T}$ of this group and then extend it to a special representation of the semidirect product $G=\mathbb{R}_{+}^{*} \wedge N$. After that, we construct the representations of the corresponding current groups $N^{X}$ and $G^{X}$ associated with $\widetilde{T}$. To describe them, instead of the classical Fock model, we use the Poisson model introduced in [11] (for brevity, we will call it simply the Poisson representation), which is more convenient for this class of groups.

The case of the Heisenberg group $N$ of order $2 n-1$ is considered separately. We describe the special representation $\widetilde{T}$ of the group $P=\mathbb{R}_{+}^{*} \wedge N$ associated with an irreducible representation $T$ of the group $N$ and the corresponding Poisson representation $U$ of the current group $P^{X}$; the representations $\widetilde{T}$ and $U$ are also irreducible. We also briefly describe, using an embedding of $P$ into the semisimple Lie group $U(n, 1)$, an extension of the special representation $\widetilde{T}$ of the group $P$ to a representation of the group $U(n, 1)$ and, correspondingly, an extension of the representation of the current group $P^{X}$ to a projective representation of the current group $U(n, 1)^{X}$ (for a detailed description, see [1] ).

The authors are grateful to N. Tsilevich for translating the paper and useful remarks.

1. Canonical families and the associated special representations of groups. We say that a unitary representation $T$ of a locally compact group $G$ in a Hilbert space $H$ is special if it has a nontrivial 1-cocycle. Recall that a 1-cocycle of $T$ is a continuous map $\beta: G \rightarrow H$ such that $\beta\left(g_{1} g_{2}\right)=T\left(g_{1}\right) \beta\left(g_{2}\right)+\beta\left(g_{1}\right)$ for any $g_{1}, g_{2} \in G$. Nontriviality means that $\beta$ cannot be written in the form $T(g) h-h$ with $h \in H$. The construction of special representations of the group $G$ (if they do exist) is based on the following notion of a canonical system of representations. 
Let $\left\{T_{r}, 0<r \leqslant r_{0}\right\}$ be a one-parameter family of unitary representations of a locally compact group $G$ in Hilbert spaces $H_{r}$ and $d m(r)$ be an infinite measure on $\left[0, r_{0}\right]$ such that $\int_{\epsilon}^{r_{0}} d m(r)<\infty$ for $\epsilon>0$. In particular, if $r_{0}<\infty$, we may take $d m(r)=r^{-1} d r$.

Definition 1. The family $\left\{T_{r}, 0<r \leqslant r_{0}\right\}$ is called a canonical family of representations with respect to the measure $m$ if the spaces $H_{r}$ do not contain nonzero vectors invariant under the operators $T_{r}(g)$ and there exist unit vectors $h_{r} \in H_{r}$ such that

$$
\int_{0}^{r_{0}}\left\|T_{r}(g) h_{r}-h_{r}\right\|_{H_{r}} d m(r)<\infty \quad \text { for every } g \in G .
$$

The family of vectors $\left\{h_{r}\right\}$ will be called almost invariant with respect to $G$.

Note that for the measure $d m(r)=r^{-1} d r$, condition (11) follows from a stronger estimate: there exists $\alpha>0$ such that

$$
\left\|T_{r}(g) h_{r}-h_{r}\right\|=O\left(r^{\alpha}\right) \text { for every } g \in G .
$$

In the definition of a canonical family, we may assume that the spaces $H_{r}$ are identified with a fixed Hilbert space $H$ so that the images of all vectors $h_{r}$ coincide with a fixed unit vector $h \in H$. Then condition (11) takes the following form.

In the space $H$, there is no nonzero vector invariant under the operators $T_{r}(g)$ and there is at least one nonzero vector $h$ such that

$$
\int_{0}^{r_{0}}\left\|T_{r}(g) h-h\right\| d m(r)<\infty \quad \text { for every } g \in G .
$$

Such a vector $h$ will be called an almost invariant vector in the space $H$. Clearly, the set of all almost invariant vectors is a linear subspace of $H$.

Remark. It follows from the definition of a canonical family that the identity representation is a limiting point for the representations $T_{r}$ as $r \rightarrow 0$ in the Fell topology on the set of unitary representations of $G$.

With every canonical family $\left\{T_{r}, 0<r<r_{0}\right\}$ of representations of a group $G$ in a Hilbert space $H$ with respect to a measure $m$ on $\left[0, r_{0}\right]$ we associate a new unitary representation $\widetilde{T}$ of this group. It is realized in the direct integral of the Hilbert spaces $H_{r}=H$ with respect to the measure $m$, i.e., in the Hilbert space

$$
\mathcal{H}=\int_{0}^{r_{0}} H_{r} d m(r), \quad H_{r}=H
$$

of sections $f(r)$ of the fiber bundle over $\left[0, r_{0}\right]$ with fibers $H_{r}, r \in\left[0, r_{0}\right]$, endowed with the norm $\|f\|^{2}=\int_{0}^{r_{0}}\|f(r)\|^{2} d m(r)$. The operators of this representation act fiberwise: $(\widetilde{T}(g) f)(r)=T_{r}(g) f(r)$.

Theorem 1. The representation $\widetilde{T}$ of the group $G$ associated with a canonical system of representations $\left\{T_{r}: 0<r<r_{0}\right\}$ is a special representation with a nontrivial 1-cocycle of the form

$$
\beta(g ; r)=T_{r}(g) h_{r}-h, \quad h_{r}=h,
$$

where $h \in H$ is an almost invariant vector in $H$. 
Indeed, it follows from the definition of a canonical family that the vector $\beta(g)$ with components $T_{r}(g) h_{r}-h_{r} \in H_{r}$ lies in the space $\mathcal{H}$ and, consequently, is a 1-cocycle of the representation $\widetilde{T}$ of the group $G$ in the space $\mathcal{H}$. The non-triviality follows from the fact that $H$ has no invariant vectors and the measure $m$ on $\left[0, r_{0}\right]$ is infinite.

Remark. It is of interest to find out the structure of representations $T$ that are limiting points in the Fell topology of the representations $T_{r}$ of a canonical system in the case where all $T_{r}$ are irreducible. If the group $G$ has no special irreducible representations (it is this case that we consider in the paper), then the only possible limit is the identity representation. If there exist irreducible special representations, then a limiting point can also coincide with one of them. It is this phenomenon that we observe in the case where $T_{r}$ is a family of complementary series representations of the semisimple Lie group $O(n, 1)$ or $U(n, 1)$, see [10].

2. The semidirect products $G=\mathbb{R}_{+}^{*} \measuredangle G_{0}$ and their special representations. In this section, we introduce a family of groups having a canonical system of representations.

Let $G_{0}$ be an arbitrary locally compact group on which an action of the group of automorphisms $\mathbb{R}_{+}^{*}$ is defined: $r: g_{0} \mapsto g_{0}^{r}$ for every $r \in \mathbb{R}_{+}^{*}$. Then

1) The group $G_{0}$ can be realized as a subgroup of the semidirect product $G=\mathbb{R}_{+}^{*} \curlywedge G_{0}$, i.e., the group of pairs $(r, g) \in \mathbb{R}_{+}^{*} \times G_{0}$ with the multiplication law

$$
\left(r_{1}, g_{1}\right)\left(r_{2}, g_{2}\right)=\left(r_{1} r_{2}, g_{1}^{r_{2}} g_{2}\right) \text {. }
$$

2) Every unitary representation $T$ of the group $G_{0}$ in a Hilbert space $K$ gives rise to a one-parameter family of unitary representations $T_{r}, r \in \mathbb{R}_{+}^{*}$, of $G_{0}$ in the same space $K$, which are given by the formula

$$
T_{r}(g)=T\left(g^{r}\right) .
$$

We say that these representations are conjugate to the original representation $T$.

Definition 2. A unitary representation $T$ of the group $G_{0}$ in a space $K$ is called summable with respect to an infinite measure $m$ on $\mathbb{R}_{+}^{*}$ with $\int_{\epsilon}^{\infty} d m(r)<\infty$ for $\epsilon>0$ if the representations $T_{r}$ of $G_{0}$ conjugate to $T$ form a canonical system with respect to $m$.

The group $G_{0}$ will be called nice if it has at least one summable representation.

In what follows, we assume that $d m(r)=e^{-\frac{u(r)}{2}} d^{*} r$, where $d^{*} r=r^{-1} d r$ is the Haar measure on $\mathbb{R}_{+}^{*}$ and $u(r)$ is a smooth positive function on the half-line such that $\int_{\epsilon}^{\infty} e^{-\frac{u(r)}{2}} d^{*} r<\infty$ for $\epsilon>0$.

Then the summability condition for a representation $T$ in a space $K$ means the nonexistence of invariant vectors $h \neq 0$ in $K$ and the existence of a nonzero vector $h \in K$ such that

$$
\int_{0}^{\infty}\left\|T_{r}(g) h-h\right\| e^{-\frac{u(r)}{2}} d^{*} r<\infty \quad \text { for every } g \in G .
$$

Theorem 1 implies the following result.

Theorem 2. Every summable unitary representation $T$ of the group $G_{0}$ in a Hilbert space $K$ gives rise to a special representation $\widetilde{T}$ of this group in the Hilbert space 
$\mathcal{K}=\int_{0}^{\infty} K_{r} d^{*} r, K_{r}=K$, which is defined by the formula $(\widetilde{T}(g) f)(r)=T_{r}(g) f(r)$. With every almost invariant vector $h \in K$ is associated the nontrivial 1-cocycle $\beta: G_{0} \rightarrow \mathcal{K}$,

$$
\beta(g, r)=e^{-\frac{u(r)}{2}}\left(T_{r}(g) h_{r}-h_{r}\right), \quad h_{r}=h .
$$

Theorem 3. The special unitary representation $\widetilde{T}$ of the group $G_{0}$ can be extended to a representation of the semidirect product $G=\mathbb{R}_{+}^{*} \wedge G_{0}$ in which the operators corresponding to elements of the subgroup $\mathbb{R}_{+}^{*}$ have the form

$$
\left(\widetilde{T}\left(r_{0}\right) f\right)(r)=f\left(r r_{0}\right) .
$$

With every almost invariant vector $h \in K$ is associated the nontrivial 1-cocycle $\beta: G \rightarrow \mathcal{K}$,

$$
\beta(g, r)=(\widetilde{T}(g) f)(r)-f(r), \text { where } f(r)=e^{-\frac{u(r)}{2}} h_{r} .
$$

Proof. The invariance of the Haar measure $d^{*} r$ implies that the operators corresponding to elements of the subgroup $\mathbb{R}_{+}^{*}$ are unitary. One can easily see that together with the operators corresponding to elements of the subgroup $G_{0}$ they generate a representation of the whole group $G$.

Further, let us verify that $\beta(g)$ is a nontrivial 1-cocycle on $G$. First of all, for $g \in G_{0}$, formula (8) coincides with (6), so that $\beta(g)$ is a nontrivial 1-cocycle on $G_{0}$. Hence it suffices to check that $\left\|\beta\left(r_{0}\right)\right\|<\infty$ for $r_{0} \in \mathbb{R}_{+}^{*}$. In this case, $\beta(g, r)=\left(e^{-\frac{u\left(r r_{0}\right)}{2}}-e^{-\frac{u(r)}{2}}\right) h_{r}$, whence $\left\|\beta\left(r_{0}\right)\right\|^{2}=\int_{0}^{\infty}\left|e^{-\frac{u\left(r r_{0}\right)}{2}}-e^{-\frac{u(r)}{2}}\right|^{2} d^{*} r<\infty$.

As we mentioned earlier, there is a subgroup $K_{0} \subset K$ such that $K_{0} \backslash 0$ is the set of all almost invariant vectors $h \in K$. Let us fix a function $u(r)=u_{0}(r)$. We can associate to each vector $h \in K_{0}$ the 1 -cocycle $\beta: G \rightarrow \mathcal{K}$ of the form (8) with $f(r)=e^{-\frac{u_{0}(r)}{2}} h$. The subset of all such cocycles forms a subgroup, wchich we denote $Z_{0}^{1}$.

Let $v: K_{0}$ onto $Z_{0}^{1}$ be the natural epimorphism. If $h, h^{\prime} \in K_{0}$, and $h-h^{\prime} \neq 0$, then $h-h^{\prime}$ is an almost invariant vector. By Theorem 3 the 1-cocycle $v(h)-v\left(h^{\prime}\right)=v\left(h-h^{\prime}\right)$ is non-cohomologous to 0 . This implies:

Corollary. Cocycles in $Z_{0}^{1}$ are pairwise non-cohomologous. The groups $K_{0}$ and $Z_{0}^{1}$ are isomorphic, $K_{0} \cong Z_{0}^{1}$.

Theorem 4. Each 1-cocycle $\beta$ of the form (8) is cohomologous to a (unique) cocycle lies in the group $Z_{0}^{1} \cong K_{0}$.

Proof. Let $u(0)=u_{0}(0)=0$, and let $f_{0}(r)=e^{\frac{-u_{0}(r)}{2}} h_{r}$. Then $f(0)=f_{0}(0)=h$. Therefore

$$
\int_{0}^{\infty}\left\|f(r)-f_{0}(r)\right\|^{2} d^{*} r<\infty
$$

So, $f(r)-f_{0}(r) \in \mathcal{K}$, and the 1-cocycle $\beta$ is cohomologous to the 1-cocycle $\beta_{0}=v(h) \in$ $Z_{0}^{1}$.

Theorem 5. If the summable representation $T$ of the group $G_{0}$ is irreducible and the representations $T_{r}$ of the group $G_{0}$ conjugate to $T$ are pairwise nonequivalent, then the special representation $\widetilde{T}$ of the group $G=\mathbb{R}_{+}^{*} \wedge G_{0}$ associated with $T$ is also irreducible.

Sometimes the representations $T_{r}$ are automatically pairwise nonequivalent; this is the case in our further examples. 
3. Special representations of nilpotent Lie groups and their one-dimensional extensions. Here we introduce a class of nilpotent Lie groups $N$ having special representations. Every group in this class can be embedded into the direct product $G=\mathbb{R}_{+}^{*} \wedge N$ and is nice in the sense of Definition 2, Note that all special representations of these groups are reducible, since irreducible representations of nilpotent groups have no nontrivial 1cocycles.

3.1. General definitions. Recall that a Lie algebra $L$ is called nilpotent of class $n$ if its lower central series $L=L_{1} \supset L_{2} \supset \cdots \supset L_{n} \supset \cdots$, where $L_{i+1}=\left[L, L_{i}\right]$, terminates at the $n$th step, i.e., $L_{n} \neq 0$ and $L_{n+1}=0$. We will say that an element $a \in L$ is of degree $r(a)=k$ if $a \in L_{k} \backslash L_{k+1}$.

The space $L$ of such an algebra can be written as the direct sum of nonzero vector spaces

$$
L=\bigoplus_{k=1}^{n} R_{k}, \quad \text { where } \quad R_{k} \subset L_{k}, \quad L_{k}=R_{k} \oplus L_{k+1} .
$$

Here every nonzero element of every space $R_{k}$ has degree $k$.

A nilpotent Lie algebra of class $n$ is called graded if there exists a decomposition of the form (9) such that

$$
\left[R_{i}, R_{j}\right] \subset R_{i+j} ; \quad R_{k}=0 \quad \text { for } \quad k>n .
$$

Each such decomposition is called a grading of the algebra $L$. In particular, all nilpotent algebras of class 1 (abelian algebras) and class 2 (metabelian algebras) are graded algebras.

The following properties are obvious:

1) Every grading of a graded algebra $L$ is uniquely determined by a subspace $R_{1} \subset L$ such that $L=R_{1} \oplus[L, L]$; the subspaces $R_{k}$ of this grading can be defined by induction on $k$ via the formula $R_{k}=\sum_{i+j=k}\left[R_{j}, R_{j}\right]$.

2) Every quotient algebra $L / L_{k}$ of a graded algebra $L$ is also graded; if $L=\bigoplus_{i=1}^{n} R_{i}$ is a grading of $L$, then $L / L_{k}=\bigoplus_{i=1}^{n-k} R_{i} / L_{k}$ is a grading of $L / L_{k}$.

Every grading (9) of a graded Lie algebra $L$ of class $n$ allows one to realize $L$ as the space of sequences $a=\left(a_{1}, \ldots, a_{n}\right), a_{k} \in R_{k}$, with the multiplication law

$$
[a, b]=\left(0, c_{2}, \ldots, c_{n}\right), \quad \text { where } \quad c_{k}=\sum_{i+j=k}\left[a_{i}, b_{j}\right] .
$$

A nilpotent Lie algebra $L$ of class $n$ is a graded algebra if and only if on $L$ there exists a diagonalizable differentiation operator $D$ with eigenvalues $1, \ldots, n$. Namely, the spaces $R_{k}$ can be defined as the eigenspaces of this operator.

Proposition 1. Every differentiation operator D with these properties gives rise to a onedimensional extension of the graded algebra $L$ to the solvable algebra $L \oplus\{D\}$ in which the commutation relations on $L$ are supplemented by the relations

$$
[D, D]=0 ; \quad[D, a]=-[a, D]=D a \quad \text { for } \quad a \in L .
$$

Remark. Examples of nongraded algebras are characteristically nilpotent algebras, in which all differentiation operators are nilpotent. 
A Lie group $N$ is called a nilpotent (graded) group of class $n$ if its Lie algebra $L$ is a nilpotent (graded) algebra of class $n$. It is known that a simply connected nilpotent group $N$ is uniquely determined by its Lie algebra $L$, and there exists a diffeomorphism $\exp : L \rightarrow N$, the exponential map, between the manifolds of this group and its Lie algebra.

If $N$ is a simply connected graded Lie group of class $n$ and (9) is a fixed grading on its Lie algebra $L$, then one can choose the coordinates of the vector space $R=\bigoplus_{k=1}^{n} R_{k}$ as coordinates on $N$. We will call them the canonical coordinates on $N$. Thus elements $a \in N$, where $N$ is a one-dimensional graded nilpotent Lie group of class $n$, are sequences of the form $a=\left(a_{1}, \ldots, a_{n}\right)$ with $a_{k} \in R_{k}$.

Theorem 6. In the canonical coordinates, the product of elements of a simply connected graded nilpotent Lie group $N$ of class $n$ has the following form:

$$
\left.a \cdot b=\left(a_{1}+b_{1}, a_{2}+b_{2}+p_{2}(a, b)\right), \ldots, a_{n}+b_{n}+p_{n}(a, b)\right),
$$

where $p_{k}(a, b) \in R_{k}$ are polynomials in $a_{1}, \ldots, a_{k-1}$ and $b_{1}, \ldots, b_{k-1}$ satisfying the condition $p_{k}(a, 0)=p_{k}(0, b)=0$. In particular, $a \cdot b=a+b+\frac{1}{2}[a, b]$ for $n=2$.

Given a graded Lie algebra $L$, the action of the differentiation $D$ on $L$ (i.e., $D a_{k}=k a_{k}$ for elements $a_{k}$ of degree $k$ ) generates an action of the group of automorphisms $\mathbb{R}_{+}^{*}$ on the graded group $N=\bigoplus_{k=1}^{n} R_{k}$ defined as $a_{k} \mapsto r^{k} a_{k}$ for $a_{k} \in R_{k}$. Thus $N$ can be extended to a solvable group $G=\mathbb{R}_{+}^{*} \wedge N$.

Remark. By a general property of solvable Lie groups, the group $G=\mathbb{R}_{+}^{*} \wedge N$ can be realized as a subgroup of the group of lower triangular matrices of a certain order $m$. In this realization, the group $\mathbb{R}_{+}^{*}$ turns into a one-dimensional subgroup of the group of diagonal matrices.

3.2. Summability of the unitary representations; main theorem. Recall that Gårding's space of the unitary representation $T$ of the Lie group $G$ in the Hilbert space $H$ is the subspace $H^{*} \subset H$, whch consists of all vectors $h \in H$ with property: $\left\langle T(g) h, h^{\prime}\right\rangle \in C^{\infty}(G)$ for all $h^{\prime} \in H$. According to the theorem by Gel'fand-Gårding, the subspace $H^{*}$ is dense in $H$.

Theorem 7. Let $N$ be an arbitrary connected and simply connected nilpotent graded Lie group and let $T$ be an arbitrary unitary representation of the group $N$ in a Hilbert space $H$. Suppose $H$ does not contain invariant vectors. Then each vector from the Garding space $H^{*} \subset H$ is almost invariant under $T$, thus the representation $T$ is summable.

Proof. Let $N \cong \bigoplus_{k=1}^{n} R_{k}, a_{k} \in R_{k}$ be a fixed graduation of the group $N$ and $a=$ $\left(a_{1}, \ldots, a_{n}\right)$ - associated with it canonical system of coordinates on $N$. Consider any vector $h \in H^{*},\|h\|=1$. Thus

$$
\langle T(a) h, h\rangle=f\left(a_{1}, \ldots, a_{n}\right) \in C^{\infty}(N), \quad f(0)=1 \quad \forall a \in N .
$$

Consequently, because of $T_{r}(a)=T\left(a^{r}\right)=T\left(r a_{1}, \ldots, r^{n} a_{n}\right)$, we obtain:

$$
\left\langle T_{r}(a) h, h\right\rangle=1+O(r) \text { for small } r .
$$

It means that, $\left\|T_{r}(a) h-h\right\|^{2} \equiv 2\left(1-\operatorname{Re}\left\langle T_{r}(a) h, h\right\rangle\right)=O(r)$. Consequently, the vector $h$ is almost invariant with respect to the representation $T$. 
Corollary. With every unitary irreducible representation $T$ of the group $N$ is associated the special irreducible representation $\widetilde{T}$ of the group $G=\mathbb{R}_{+}^{*}<N$.

4. The special representation of the group $\mathbb{R}_{+}^{*} \wedge N$ associated with the regular representation of $N$. The regular representation of a locally compact group $G$ is the unitary representation of $G$ in the Hilbert space $L^{2}(G, d g)$, where $d g$ is the right-invariant measure on $G$, by right translations: $\left(T\left(g_{0} f\right)(g)\right)=f\left(g g_{0}\right)$.

Let us describe the structure of the regular representation of a connected, simply connected nilpotent graded Lie group $N$ of class $n$. Let

$$
N=\bigoplus_{i=1}^{n} R_{i}
$$

be a fixed grading of $N$. With this grading, elements of $N$ can be written as sequences $a=\left(a_{1}, \ldots, a_{n}\right), a_{i} \in R_{i}$, and the right-invariant measure on $N$ is $d a=d a_{1} \cdots d a_{n}$, where $d a_{i}$ is the Lebesgue measure on the vector space $R_{i}$.

In our notation, the regular representation $T$ of the group $N$ is a representation in the Hilbert space $K=L^{2}(N, d a)$ of functions $f(a)=f\left(a_{1}, \ldots, a_{n}\right)$ with the norm $\left.\|f\|\right|^{2}=$ $\int_{N}|f(a)|^{2} d a$. The operators of this representation are defined by the formula

$$
(T(b) f)(a)=f(a \cdot b), \quad b \in N,
$$

where $a \cdot b$ is given by (12).

The operators of the representations $T_{r}$ conjugate to $T$ have the form

$$
\left(T_{r}(b) f\right)(a)=f\left(a \cdot b^{r}\right), \quad b \in N, \quad \text { where } \quad b^{r}=\left(r b_{1}, r^{2} b_{2}, \ldots, r^{n} b_{n}\right) .
$$

It is clear that the space $K$ does not contain vectors invariant under the operators of $T$, but, by Theorem 7 it contain almost invariant vectors.

Let us describe a family of almost invariant vectors $F_{\mu}$ in the space $K$ of the representation $T$. Let $\mu=\left(\mu_{1}, \ldots, \mu_{n}\right)$ be an arbitrary sequence of positive definite quadratic forms $\mu_{i}: R_{i} \rightarrow \mathbb{R}$. We assign to $\mu$ a function $F_{\mu}$ on $N$ :

$$
F_{\mu}(a)=\exp (-\mu(a))=\exp \left(-\sum_{i=1}^{n} \mu_{i}\left(a_{i}\right)\right),
$$

where $a=\left(a_{1}, \ldots, a_{n}\right), a_{i} \in R_{i}$. It is evident that $F_{\mu} \in K$, and the linear space spanned by such vectors is dense in $K$.

Theorem 8. All vectors $F_{\mu}$ of the form (13) are almost invariant in the space of the regular representation of the graded nilpotent group $N$.

Proof. We fix a sequence $\mu=\left(\mu_{1}, \ldots, \mu_{n}\right)$. Let $F_{k}(a)=\exp \left(-\sum_{i=1}^{k} \mu_{i}\left(a_{i}\right)\right), k=1, \ldots, n$. In particular, $F_{n}(a)=F_{\mu}(a)$. Consider $F_{k}(a)$ as a function on the group $N / N_{k+1}$, where $N_{i+1}=\left[N, N_{i}\right]$. Then $F_{k}(a)$ lies in $H_{k}$, the space of the regular representation of $N / N_{k+1}$.

We may assume that the Lebesgue measures $d a_{i}$ on $R_{i}$ are normalized so that $\int_{R_{i}} e^{-2 \mu_{i}\left(a_{i}\right)} d a_{i}=1$. Then $\left\|F_{k}\right\|=1$, and the almost invariance condition (50) for the vector $F_{k} \in H_{k}$ with respect to the measure $d m(r)=e^{-\frac{u(r)}{2}} d^{*} r$ is equivalent to

$$
\int_{0}^{1}\left(1-\left\langle T_{r}(b) F_{k}, F_{k}\right\rangle\right)^{1 / 2} d^{*} r<\infty \quad(k=1, \ldots, n) .
$$


We will prove, by induction on the class $n$, the following estimate:

$$
\left\langle T_{r}(b) F_{n}, F_{n}\right\rangle=1+O\left(r^{2}\right) \text { on }(0,1) .
$$

Obviously, condition (14) immediately follows from this estimate.

First note that the representations $T_{r}, r \in \mathbb{R}_{+}^{*}$, of $N$ conjugate to the regular representation $T$ have the form

$$
\left(T_{r}(b) f\right)(a)=f\left(a \cdot b^{r}\right), \quad b \in N, \quad \text { where } \quad b^{r}=\left(r b_{1}, r^{2} b_{2}, \ldots, r^{n} b_{n}\right) .
$$

By Theorem [6, for every fixed $b$ this formula can be written as

$$
\left(T_{r}(b) f\right)(a)=f\left(a_{1}+r b_{1}, a_{2}+r q_{2}(a, r, b), \cdots, a_{n}+r q_{n}(a, r, b)\right),
$$

where $q_{k}(a, r, b) \in R_{k}$ are polynomials in $a_{1}, \ldots a_{k-1}$ and $r, b_{1}, \ldots, b_{k}$. In what follows, instead of $q_{k}(a, r, b)$ we will write $q_{k}(a)$ or $q_{k}\left(a_{1}, \ldots, a_{k-1}\right)$.

In the case $n=1$, we have

$$
\left\langle T_{r}(a) F_{1}, F_{1}\right\rangle=\int_{R_{1}} e^{-\mu_{1}\left(a_{1}+r b_{1}\right)-\mu_{1}\left(a_{1}\right)} d a_{1}=e^{-\frac{r^{2}}{2} \mu\left(b_{1}\right)} .
$$

Here $\langle\cdot, \cdot\rangle$ is the scalar product on $H_{1}$. This immediately implies (15) for $n=1$.

Let us prove (15) for an arbitrary $n$ under the assumption that it is proved for $n-1$. We use the relation

$$
F_{n}\left(a_{1}, \ldots, a_{n}\right)=F_{n-1}\left(a_{1}, \ldots, a_{n-1}\right) e^{-\mu_{n}\left(a_{n}\right)} .
$$

It implies that

$$
\left\langle T_{r}(b) F_{n}, F_{n}\right\rangle=\int_{N}\left(\left(T_{r}(b) F_{n-1}\right)(a) F_{n-1}(a) e^{-\mu_{n}\left(a_{n}+r q_{n}(a)\right)-\mu_{n}\left(a_{n}\right)}\right) d a_{1} \cdots d a_{n} .
$$

Here $\langle\cdot, \cdot\rangle$ is the scalar product on $H_{n}$. Integrating with respect to $a_{n}$ and observing that only the last factor in the integrand depends on $a \cdot n$, we obtain

$$
\left.\left\langle T_{r}(b) F_{n}, F_{n}\right\rangle=\int_{N}\left\langle T_{r}(b) F_{n-1}(a), F_{n-1}(a)\right\rangle\right) e^{-\frac{r^{2}}{2} \mu_{n}\left(q_{n}(a)\right)} d a_{1} \cdots d a_{n-1} .
$$

Therefore,

$$
\left\langle T_{r}(b) F_{n}, F_{n}\right\rangle=\left\langle T_{r}(b) F_{n-1}, F_{n-1}\right\rangle-r^{2} \int\left(Q(a)\left\langle T_{r}(b) F_{n-1}(a), F_{n-1}(a)\right\rangle\right) d a_{1} \cdots d a_{n-1},
$$

where $Q(a)$ is a bounded function of $r$ and $a_{1}, \ldots a_{n-1}$. Since $Q(a)$ is bounded, the function $I(r)=\int\left(Q(a)\left\langle T_{r}(b) F_{n-1}, F_{n-1}\right\rangle\right) d a_{1} \cdots d a_{n-1}$ is bounded on [0,1]. Hence, in view of (17), estimate (14) for $F_{n-1}$ implies the same estimate for $F_{n}$.

Corollary. The special representation $\widetilde{T}$ of the group $G=\mathbb{R}_{+}^{*} \wedge N$, associated with regular representation $T$ of the group $N$, has a family of nontrivial 1-cocycles of type

$$
\beta_{\mu}(g, r)=\left(\widetilde{T}(g) f_{\mu}\right)(r)-f_{\mu}(r), \text { where } f_{\mu}(r)=e^{-\frac{r}{2}} F_{\mu}
$$

parametrized by sequences $\mu=\left(\mu_{1}, \ldots, \mu_{n}\right)$ of positive definite quadratic forms $\mu_{i}: R_{i} \rightarrow \mathbb{R}$. By Theorem 3 (corollary) these cocycles $\beta_{\mu}$ are pairwise non-cohomologous. 
5. The case of the Heisenberg group. Here we describe the special representation of the Heisenberg group $N$ of order $2 n-1$ associated with an irreducible representation of $N$ and its extension to a representation of the group $P=\mathbb{R}_{+}^{*}<N$.

The group $N$ is a nilpotent group of class 2 , so that we can apply the above definitions and constructions. We realize $N$ as the set of pairs $(i t, z), t \in \mathbb{R}, z \in \mathbb{C}^{n-1}$, or, which is more convenient, as the set of pairs

$$
(\zeta, z), \quad \text { where } \quad \zeta=i t-\frac{1}{2}|z|^{2}, \quad t \in \mathbb{R}, \quad z \in \mathbb{C}^{n-1},
$$

with the multiplication law

$$
\left(\zeta_{1}, z_{1}\right)\left(\zeta_{2}, z_{2}\right)=\left(\zeta_{1}+\zeta_{2}-z_{1} z_{2}^{*}, z_{1}+z_{2}\right) .
$$

The group of automorphisms $\mathbb{R}_{+}^{*}$ acts on $N$ according to the formula $(\zeta, z) \mapsto\left(r^{2} \zeta, r z\right)$, so that we have an embedding of $N$ into the semidirect product $P=\mathbb{R}_{+}^{*} \wedge N$.

Up to conjugacy of representations with respect to the action of the group $\mathbb{R}_{+}^{*}$, there are two irreducible infinite-dimensional unitary representations of the group $N$. The first representation $T$ can be realized in the Hilbert space $K$ of entire analytic functions $f(z)$ on $\mathbb{C}^{n-1}$ with the norm

$$
\|f\|^{2}=\int_{\mathbb{C}^{n-1}}|f(z)|^{2} e^{-z z^{*}} d \mu(z), \quad z z^{*}=\sum z_{i} \bar{z}_{i}
$$

its operators have the form

$$
\left(T\left(\zeta_{0}, z_{0}\right) f\right)(z)=e^{\zeta_{0}-z z_{0}^{*}} f\left(z+z_{0}\right) .
$$

The second representation can be realized in the space of entire antianalytic functions. For definiteness, we confine ourselves to considering the first representation $T$.

The special representation $\widetilde{T}$ of the group $N$ associated with $T$ is realized in the space $\mathcal{K}=\int_{0}^{\infty} K_{r} d^{*} r$ and is given by the formula

$$
\left(\widetilde{T}\left(\zeta_{0}, z_{0}\right) f\right)(r, z)=e^{r^{2} \zeta_{0}-r z z_{0}^{*}} f\left(r, z+r z_{0}\right) .
$$

It can be extended to a special unitary representation of the group $P=\mathbb{R}_{+}^{*} \wedge N$, where the operators corresponding to elements of the subgroup $\mathbb{R}_{+}^{*}$ are given by the formula

$$
\left(\widetilde{T}\left(r_{0}\right) f\right)(r, z)=f\left(r r_{0}, z\right) .
$$

The representation $\widetilde{T}$ of the group $P$ is irreducible and has a nontrivial 1-cocycle of the form

$$
b(g ; r, z)=(\widetilde{T}(g) f)(r, z)-f(r, z), \quad \text { where } f(r, z)=e^{-r^{2}} .
$$

There exists an embedding of $P$ into the semisimple Lie group $U(n, 1)$ such that every element $g \in U(n, 1)$ can be uniquely written as a product $g=p u, p \in P, u \in U$, where $U$ is the maximal compact subgroup of $U(n, 1)$. Using this embedding, we briefly describe the extension of the special representation $\widetilde{T}$ of the group $P$ to a special unitary representation of the group $U(n, 1)$ (for more details, see [11]).

Consider the homogeneous space $L=U \backslash U(n, 1)$ of the group $U(n, 1)$ realized as the space of pairs $v=(a, b) \in \mathbb{C} \oplus \mathbb{C}^{n-1}$, where $a+\bar{a}+b b^{*}<0$. The subgroup $U$ is the stationary subgroup of the point $v_{0}=(-1,0)$, so that every element $v \in L$ can be written in the form $v=p v_{0}$ with $p \in P$. 
With every point $v=(a, b) \in L$ we associate the following function $f_{v}$ on $\mathbb{R}_{+}^{*} \times \mathbb{C}^{n-1}$ :

$$
f_{v}(r, z)=e^{r^{2} a+(z, b)}, \quad \text { where } \quad(z, b)=\sum z_{i} b_{i} .
$$

For every fixed $r$, these functions lie in the space $K$ and form a total subset in $K$; their differences $f_{v_{1}}-f_{v_{2}}$ lie in the space $\mathcal{K}=\int_{0}^{\infty} K_{r} d^{*} r$ and form a total subset $M$ in $\mathcal{K}$.

On the set $M$, the original formulas for the operators $\widetilde{T}(g)$ of the special representation of $P$ are equivalent to the following ones:

$$
\widetilde{T}(g)\left(f_{v_{1}}-f_{v_{2}}\right)=f_{g v_{1}}-f_{g v_{2}} .
$$

Definition 3. The extension of the representation $\widetilde{T}$ of the subgroup $P$ to the group $U(n, 1)$ is defined on the total set $M$ by the same formula (24).

The nontrivial 1-cocycle of this representation has the form

$$
b(g)=f_{g v_{0}}-f_{v_{0}} .
$$

6. The Poisson representations of current groups $N^{X}$, where $N$ is a graded nilpotent Lie group. We begin with brief definitions of the countable tensor product of Hilbert spaces and quasi-Poisson measures.

The countable tensor product $\otimes_{n=1}^{\infty}\left(K_{n}, h_{n}\right)$ of punctured Hilbert spaces $\left(K_{n}, h_{n}\right)$, where $h_{n} \in K_{n}$ are fixed unit vectors in Hilbert spaces $K_{n}$, is the Hilbert space obtained by the norm completion of the inductive limit of the finite tensor products $H_{k}=\otimes_{n=1}^{k} K_{n}$ with respect to the embeddings $H_{k} \rightarrow H_{k} \otimes h_{k+1} \subset H_{k+1}$.

The Poisson and quasi-Poisson measures. Every pair $(Y, \mu)$, where $Y$ is a standard Borel space (the state space) and $\mu$ is an infinite $\sigma$-finite continuous measure on $Y$ (the intensity measure) gives rise to the Poisson pair $(\mathcal{E}(Y), \nu)$, where $\mathcal{E}(Y)$ is the space of all configurations on $Y$ (finite or countable collections of points from $Y$ containing every point with at most finite multiplicity) and $\nu$ is the Poisson measure on $\mathcal{E}(Y)$ with intensity measure $\mu$; for more details, see [6] and [11].

A quasi-Poisson measure associated with the Poisson measure $\nu$ is an infinite (sigmafinite) measure $\sigma$ on $\mathcal{E}(Y)$ that is equivalent with respect to $\nu$; for more details, see [11].

In what follows, we assume that $(Y, \mu)=\left(\mathbb{R}_{+}^{*}, e^{-u(r)} d^{*} r\right) \times(X, m)$, where $(X, m)$ is a space with a probability measure $m$ and $u(r)$ is a fixed positive function on the half-line such that $\int_{\epsilon}^{\infty} e^{-\frac{u(r)}{2}} d^{*} r<\infty$ for $\epsilon>0$. Thus $\mathcal{E}(Y)$ is the space of countable sequences $\omega=\left\{\left(r_{i}, x_{i}\right)\right\}$, where $r_{i} \in \mathbb{R}_{+}^{*}, X_{i} \in X$, and $\nu$ is the Poisson measure on $\mathcal{E}(Y)$ with intensity measure $e^{-u(r)} d^{*} r d m(x)$. In what follows, we assume that $\sigma$ is the quasi-Poisson measure $\sigma$ associated with $\nu$ that is given by the formula

$$
d \sigma(\omega)=\pi(\omega) d \nu(\omega), \quad \text { where } \pi(\omega)=e^{-\sum_{(r, x) \in \omega} u(r)} .
$$

The measure $\sigma$ is uniquely determined by its characteristic functional, which has the following form:

$$
\int_{\mathcal{E}(Y)} \exp \left(-\sum_{y \in \omega} f(y)\right) d \sigma(\omega)=\exp \left(\int_{\mathbb{R}_{+}^{*} \times X}\left(e^{-f(r, x)}-e^{-u(r)} d^{*} r d m(x)\right) .\right.
$$

Now we describe the quasi-Poisson representation of the current group $N^{X}$ associated with a special representation $T$ of the group $N$. Let $K$ be the space of the representation $T$ 
and $h \in K$ be an almost invariant vector of $T$. With every configuration $\omega \in \mathcal{E}(Y)$ we associate the countable tensor product of punctured Hilbert spaces $\left(K_{y}, h_{y}\right)=(K, h)$ :

$$
K_{\omega}^{\otimes}=\bigotimes_{(r, x) \in \omega} K_{r, x}, \quad K_{r, x}=K .
$$

The representation $U$ of the group $N^{X}$ associated with the representation $T$ of the group $N$ can be realized in the Hilbert space

$$
\operatorname{QPS}\left(\mathcal{E}(Y), \sigma, K_{\omega}^{\otimes}\right)
$$

of sections $f(\omega)$ of the fiber bundle $\mathcal{E}(Y)$ with fibers $K_{\omega}^{\otimes}, \omega \in \mathcal{E}(Y)$, endowed with the norm

$$
\|f\|^{2}=\int_{\mathcal{E}(Y)}\|f(\omega)\|^{2} d \sigma(\omega) .
$$

The operators of this representation are defined on the set of vectors of the form $F_{v}(\omega)=$ $\otimes_{(r, x) \in \omega} v(r, x)$ (which is total in $\operatorname{QPS}\left(\mathcal{E}(Y), \sigma, K_{\omega}^{\otimes}\right)$ ) by the following formula:

$$
\left(U(g(\cdot)) F_{v}\right)(\omega)=\bigotimes_{(r, x) \in \omega} T_{r}(g(x)) v(r, x),
$$

where $T_{r}$ are the representations of $N$ conjugate to the special representation $T$.

The representation $U$ of the group $N^{X}$ is obviously reducible.

7. The extension of the representation $U$ of the group $N^{X}$ to a representation of the group $G^{X}$, where $G=\mathbb{R}_{+}^{*} \wedge N$. Now we describe an extension of the representation $U$ of the group $N^{X}$ in the quasi-Poisson space $\operatorname{QPS}\left(\mathcal{E}(Y), \sigma, K_{\omega}^{\otimes}\right)$ constructed above to a representation of the group $G^{X}=\left(\mathbb{R}_{+}^{*}\right)^{X} \wedge N^{X}$, where, by definition, $\left(\mathbb{R}_{+}^{*}\right)^{X}$ is the group of measurable maps $\widetilde{r}: X \rightarrow \mathbb{R}_{+}^{*}$ such that

$$
\int_{X}|\log r(x)| d m(x)<\infty
$$

There is a natural action of the group $\left(\mathbb{R}_{+}^{*}\right)^{X}$ by transformations

$$
(r, x) \mapsto \widetilde{r}(r, x)=(r(x) r, x)
$$

of the space $Y=\mathbb{R}_{+}^{*} \times X$ and, consequently, by transformations $\omega \mapsto \widetilde{r} \omega$ of the space of configurations $\mathcal{E}(Y)$. It is known (see [11]) that the quasi-Poisson measure $\sigma$ is ergodic and projectively invariant under the group of transformations $\left(\mathbb{R}_{+}^{*}\right)^{X}$ :

$$
d \sigma(\widetilde{r} \omega)=e^{\int_{X} \log r(x) d m(x)} d \sigma(\omega) .
$$

With the elements of the group $\left(\mathbb{R}_{+}^{*}\right)^{X}$ we associate the operators on $\operatorname{QPS}\left(\mathcal{E}(Y), \sigma, K_{\omega}^{\otimes}\right)$ given by the formula

$$
(U(\widetilde{r}) f)(\omega)=e^{\frac{1}{2} \int_{X} \log r(x) d m(x)} f(\widetilde{r} \omega) .
$$

Theorem 9. The operators $U(\widetilde{r})$ are unitary; together with the operators corresponding to the elements of the subgroup $N^{X}$, they generate a unitary representation of the whole group $G^{X}$. 
Indeed, the operators $U(\widetilde{r})$ are unitary by the projective invariance of the measure $\sigma$. The second assertion immediately follows from the definition of these operators.

Note that the operators $U(g)$ for the whole group $G^{X}$ can be defined by a single formula:

$$
\left(U(g) F_{v}\right)(\omega)=e^{\frac{1}{2} \int_{X} \log \widetilde{r}(x) d m(x)} F_{\widetilde{T}(g) v},
$$

where $r(\cdot)$ is the image of $g(\cdot)$ under the homomorphism $G^{X} \rightarrow\left(\mathbb{R}_{+}^{*}\right)^{X}$.

Theorem 10. If the summable representation $T$ of the group $N$ satisfy to the conditions of Theorem 5, where $G_{0}=N$, then the representation $U$ of the group $G^{X}=\mathbb{R}_{+}^{*} \wedge N^{X}$ in the space $\mathrm{QPS}\left(\mathcal{E}(Y), \sigma ; K_{\omega}^{\otimes}\right)$ associated with $T$ is irreducible.

Proof. Let us prove that every bounded operator $A$ on $\operatorname{QPS}\left(\mathcal{E}(Y), \sigma ; K_{\omega}^{\otimes}\right)$ that commutes with the operators $U(g), g \in G^{X}$, is a scalar operator.

It follows from Theorem 5 that the representations $U_{\omega}(g)$ of the group $N^{X}$ in the spaces $K_{\omega}^{\otimes}$ are (up to a subset of zero measure in the space $\mathcal{E}(Y)$ ) irreducible and pairwise nonequivalent. Hence, every operator $A$ on $\operatorname{QPS}\left(\mathcal{E}(Y), \sigma ; K_{\omega}^{\otimes}\right)$ that commutes with the operators $U(g), g \in N^{X}$, is scalar on every $K_{\omega}^{\otimes}$. i.e., is the multiplication by a function $f(\omega)$. If $A$ commutes also with the operators $U(g), g \in\left(\mathbb{R}_{+}^{*}\right)^{X}$, then $f(\omega)$ is constant on the orbits of this group un the space $\mathrm{E}(Y)$. Since the measure $\sigma$ is ergodic with respect to $\left(\mathbb{R}_{+}^{*}\right)^{X}$, it follows that $A$ is a scalar operator.

8. The Poisson representation of the current group $P^{X}=\left(\mathbb{R}_{+}^{*}\right)^{X} \wedge N^{X}$, where $N$ is the Heisenberg group of order $2 n-1$, and its extension to a representation of the current group $U(n, 1)^{X}$. Let $T$ be the irreducible representation of the Heisenberg group $N$ in the Hilbert space $K$ described in Section [5] and $\sigma$ be the quasi-Poisson measure on $\mathcal{E}(Y)=\mathcal{E}\left(\mathbb{R}_{+}^{*} \times X\right)$ with characteristic functional (25), where $u(r)-2 r^{2}$.

We will describe the representation $U$ of the current group $P^{X}$ in the quasi-Poisson space $\operatorname{QPS}\left(\mathcal{E}(Y), \sigma, K_{\omega}^{\otimes}\right)$ associated with $T$.

Let $\widetilde{T}$ be the special representation of the group $P$ associated with $T$, see Section 5 , Consider the following total subset of vectors in $\operatorname{QPS}\left(\mathcal{E}(Y), \sigma, K_{\omega}^{\otimes}\right)$ :

$$
F_{p}(\omega)=F_{\widetilde{T}(p) f}=\bigotimes_{(r, x) \in \omega}(\widetilde{T}(p(x)) f)(r, x ; z), \quad \text { where } \quad f(r, x ; z)=e^{-r^{2}}
$$

and $p$ ranges over the group $P^{X}$. By the general formula (27), the operators $U(p)$ for elements of the group $P^{X}$ act on this set according to the formula

$$
U\left(p_{0}\right) F_{p}=e^{\frac{1}{2} \int_{X} \log r_{0}(x) d m(x)} F_{p_{0} p}
$$

where $r_{0}(\cdot)$ is the image of $p_{0}(\cdot)$ under the homomorphism $P^{X} \rightarrow \mathbb{R}_{+}^{*}$.

It follows from the irreducibility of $T$ that the representation $U$ of the current group $P^{X}$ is also irreducible.

According to Section $[5$, the special representation $\widetilde{T}$ of the group $P$ can be extended to a representation of the group $U(n, 1)$. Using this extension, we will describe an extension of the representation of $P^{X}$ to a representation of $U(n, 1)^{X}$.

In the space $\operatorname{QPS}\left(\mathcal{E}(Y), \sigma, K_{\omega}^{\otimes}\right)$ we consider the set $M$ of vectors of the form

$$
F_{g}(\omega)=\bigotimes_{(r, x) \in \omega}(\widetilde{T}(g(x)) f)(r, x ; z), \quad g \in U(n, 1)^{X} .
$$


Note that $F_{g u}=F_{g}$ for every $u \in U^{X}$. Thus $M$ coincides with the total set (28) of vectors $F_{p}, p \in P^{X}$.

We define the operators $U\left(g_{0}\right)$ for $g_{0} \in U(n, 1)^{X}$ on the set $M$ by the following formula:

$$
U\left(g_{0}\right) F_{g}=e^{\int_{X} l \lambda\left(g_{0}(x), g(x)\right) d m(x)} F_{g_{0} g},
$$

where

$$
\lambda\left(g_{0}, g\right)=-\frac{1}{2}\left\|b\left(g_{0}\right)\right\|^{2}-\operatorname{Re}\left\langle b\left(g_{0}\right), f\right\rangle-\left\langle\widetilde{T}\left(g_{0}\right) b(g), \widetilde{T}\left(g_{0}\right) f\right\rangle+\langle b(g), f\rangle .
$$

The restrictions to $P^{X}$ of the operators $U(g)$ thus defined coincide on $M$ with the operators $U(p)$ of the representation of the group $P^{X}$ defined above.

Theorem 11. The operators $U(g), g \in U(n, 1)^{X}$, preserve the inner products on $M$ and are related by the formula

$$
U\left(g_{1} g_{2}\right) F_{g}=e^{i \int_{X} \rho\left(g_{1}(x), g_{2}(x)\right) d m(x)} U\left(g_{1}\right) U\left(g_{2}\right) F_{g} \quad \text { for any } \quad g_{1}, g_{2}, g \in U(n, 1)^{X},
$$

where

$$
\rho\left(g_{1}, g_{2}\right)=-\operatorname{Im}\left(\left\langle b\left(g_{2}\right), b\left(g_{1}^{-1}\right\rangle\right)\right)+\operatorname{Im}\left\langle b\left(g_{1} g_{2}\right)-b\left(g_{1}\right)-b\left(g_{2}\right), f\right\rangle .
$$

Therefore, they induce an extension of the original representation of the group $P^{X}$ to a unitary projective representation of the group $U(n, 1)^{X}$.

\section{References.}

[1] H. Araki, Factorisable representation of current algebra, Publ. Res. Inst. Math. Sci. 5 (1969/1970), $361-422$.

[2] B. Bekka, P. de la Harpe, and A. Valette, Kazhdan Property (T), Cambridge Univ. Press, Cambridge, 2008.

[3] I. M. Gelfand, M. I. Graev, and A. M. Vershik, Models of representations of current groups, in: Representations of Lie Groups and Lie Algebras, Akad. Kiado, Budapest, 1985, pp. 121-179.

[4] V. V. Gorbatsevich, A. L. Onishchik, and E. B. Vinberg, Foundations of Lie Theory and Lie Transformation Groups, Springer-Verlag, Berlin, 1997.

[5] R. S. Ismagilov, Representations of Infinite-Dimensional Groups, Transl. Math. Monogr., Vol. 152, Amer. Math. Soc., Providence, RI, 1996.

[6] J. F. C. Kingman, Poisson Processes, The Clarendon Press, Oxford Univ. Press, New York, 1993.

[7] A. A. Kirillov, Unitary representations of nilpotent Lie groups, Uspekhi Mat. Nauk 17, No. 4 (1962), $57-110$.

[8] Yu. A. Neretin, Categories of Symmetries and Infinite-Dimensional Groups, The Clarendon Press, Oxford Univ. Press, New York, 1996.

[9] A. M. Vershik, I. M. Gelfand, and M. I. Graev, Irreducible representations of the group $G^{X}$ and cohomologies, Funct. Anal. Appl. 8, No. 2, 151-153 (1974).

[10] A. M. Vershik and M. I. Graev, The structure of complementary series and special representations of the groups $O(n, 1)$ and $U(n, 1)$, Russian Math. Surveys 61, No. 5 (2006), 799-884.

[11] A. M. Vershik and M. I. Graev, The Poisson model of the Fock space and representations of current groups, Algebra i Analiz 23, No. 3 (2011), 63-136.

[12] A. M. Vershik and S. I. Karpushev, Cohomology of groups in unitary representations, the neighborhood of the identity, and conditionally positive definite functions, Math. USSR-Sb. 47, No. 2 (1984), 513-526.

A. M. Vershik, St. Petersburg Department of Steklov Institute of Mathematics, 27 Fontanka, St. Petersburg 191023, Russia.

E-mail address: vershik@pdmi.ras.ru 
M. I. Graev, Institute for System Studies, 36-1 Nakhimovsky Pr., 117218 Moscow, Russia. E-mail address: graev_36@mtu-net.ru 